\title{
Using Tsypkin's Approach for the Study of a Class of Mixed-Signal Nonlinear Systems
}

\author{
Jérôme Juillard and Eric Colinet
}

\begin{abstract}
An extension of Tsypkin's approach is proposed, in order to determine the values of the oscillation frequencies of mixed-signal nonlinear systems consisting of one possibly nonideal relay, one discrete-time block with a zeroth-order hold, and one continuous-time block. Several methods for determining the shape of the complex oscillations in some situations are then proposed and discussed.
\end{abstract}

Index Terms-Limit cycles, mixed-signal systems, relay feedback systems.

\section{INTRODUCTION}

$\mathbf{R}$ ESONANT microelectromechanical systems (MEMS) have been a growing field of interest [1] for the last few years. The basic principle of resonant MEMS is to measure the frequency shift of an oscillating microstructure that is affected by the variation of a given physical quantity (such as acceleration [2] or pressure [3]). Other resonant MEMS in which the frequency of the oscillations is also a parameter of interest include microgyroscopes [4] and micromechanical bandpass filters [5], [6]. In all those applications, the microstructure is usually brought to oscillate via a feedback scheme [2], [7], [8] that may be composed of analog and digital components. In many publications, the analysis of the system is performed using describing function methods and neglecting the influence of sampling and holding on the performance of the system.

While this approximation is satisfactory for a rough estimation of the behaviour of the system, it falls down when, as in our case, a more precise analysis is required. For example, consider the system of Fig. 1. Whenever the mass passes its position of equilibrium, a Dirac impulse (the first derivative of the relay's output) is delivered to the system, which is therefore excited. It can be shown [9] that the oscillation frequency of such a system is $\omega_{0} \sqrt{1-\xi^{2}}$. Such a feedback scheme may be very useful in various MEMS applications, notably those involving electrostatic actuation [10]. However, realizing such a system will be very impractical, and solutions like that of Fig. 2, which involve discrete-time components, will usually be preferred. It

Manuscript received October 8, 2004; revised June 16, 2005, November 14, 2005 , and January 10, 2006. This paper was recommended by L. Trajkovic.

J. Juillard is with the Department of Signal Processing and Electronic Systems, SUPELEC, 91192 Gif-sur-Yvette Cedex, France (e-mail: jerome.juillard@supelec.fr).

E. Colinet was with the Department of Signal Processing and Electronic Systems, SUPELEC, 91192 Gif-sur-Yvette Cedex, France. He is now with French Atomic Energy Commission, Laboratory of Microelectronics and Technology for Information (CEA-LETI), 38054 Grenoble Cedex 9, France (e-mail: eric.colinet@cea.fr).

Digital Object Identifier 10.1109/TCSI.2006.883862

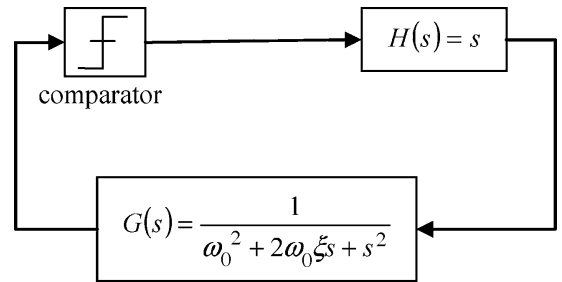

Fig. 1. Mass-spring-damper system $G(s)$ with natural pulsation $\omega_{0}$ and damping coefficient $\xi$ can be brought to oscillate by adding a relay and a continuous differentiator $H(s)$ in its feedback path. $H(s)$ ideally delivers Dirac impulses.

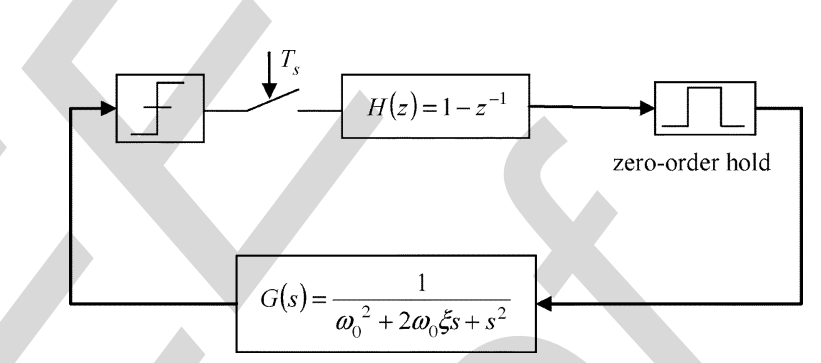

Fig. 2. Discrete-time differentiator delivers pulses of finite intensity and duration to $G(s)$.

is quite clear that the frequency of the oscillations will be affected by the value of the sampling period of the discrete part (which is equal to the duration of the delivered pulses).

The analysis of such a mixed-signal nonlinear system is no longer trivial and, to the best of our knowledge, there currently exists no simple method for determining precisely its oscillation frequency. Although there exists some abundant literature on the subject of oscillations of relay feedback systems [11]-[14], most of it deals either with solely continuous systems or with solely discrete ones. Also, many of these studies emerge from a context of control applications. From this perspective, oscillations are at best parasitic and must be avoided at all costs. As a consequence, they are mostly dedicated to showing the existence of periodic solutions and studying their stability. This is not so in the context of resonant sensors: a sustained oscillation must be obtained for the sensor to work properly. For some applications [15], the shape of the limit cycle may also be of great importance. The determination of this shape has been studied extensively in the context of first and second-order sigma-delta converters, as in [16] or [17], but not from a more general point of view. The present work aims at filling some of the gaps that are left in the study of the oscillations of relay feedback systems.

In this paper, we propose using Tsypkin's approach [18] in order to determine the limit cycles of mixed-signal nonlinear systems consisting of:

- one possibly nonideal relay;

- one discrete-time block with a zeroth-order hold; 


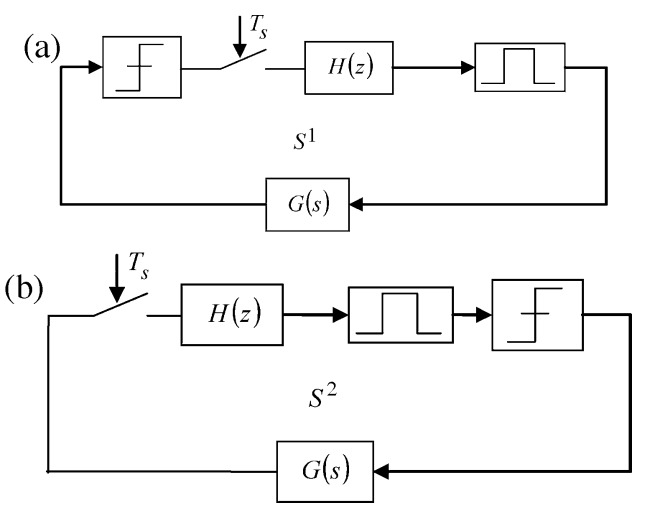

Fig. 3. Tsypkin's approach may be extended to both systems $S^{1}$ (top) and $S^{2}$ (bottom).

- one continuous time block.

These systems are represented in Fig. 3.

We first briefly restate Tsypkin's method for determining the oscillation frequencies of continuous nonlinear systems. Then, we show how to extend this method to the case of the simple and complex oscillations of mixed-signal systems. A method for determining the shape of the complex oscillations is given and illustrated and its implementation is discussed.

\section{EXTENSION OF TSYPKIN's METHOD TO MiXED-SignAL NONLINEAR SYSTEMS}

\section{A. Tsypkin's Method Applied to Continuous Systems}

Let us consider a system $S^{c}$ consisting of a possibly nonideal relay and a continuous time system, as in Fig. 4. Tsypkin's method may be used in order to determine the existence of possible limit cycles of $S^{c}$ and their characteristics (pulsation and amplitude). In the case of simple oscillations of the system, the output $s(t)$ of the relay is a square wave with period $T_{0}$. Tsypkin's criterion, which is closely related to Hamel's [19], consists in saying that $S^{c}$ oscillates with period $T_{0}$ if the relay's switching conditions are met periodically. Taking the origin of time on a rising edge of the relay, these switching conditions may be expressed as

$$
\left\{\begin{array}{l}
\varepsilon\left(\frac{T_{0}}{2}\right)=0 \\
\frac{d \varepsilon}{d t}\left(\frac{T_{0}}{2}\right)<0
\end{array}\right.
$$

where $\varepsilon(t)$ is the relay's input. Verifying (1) for a given $T_{0}$ then becomes a simple matter of:

- expressing the relay's output $s(t)$ as a Fourier series;

- expressing the time response $\varepsilon(t)$ of $G(s)$ to this input, also as a Fourier series;

- evaluating this time response and its first derivative for $t=$ $\left(T_{0} / 2\right)$.

There usually exists no analytical solution to (1); the oscillation criterion must then be checked in a graphical way, for example, by plotting (2) in the complex plane

$$
\Lambda(T)=\varepsilon\left(\frac{T}{2}\right)+j \frac{d \varepsilon}{d t}\left(\frac{T}{2}\right)
$$

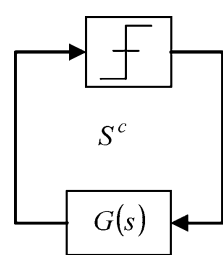

Fig. 4. Tsypkin's approach is usually applied to systems consisting of one relay and one continuous block.

and finding its intersection with the real axis. For the differentiator system of Fig. 1, $\Lambda(T)$ is given in Fig. 5 for $\omega_{0}=1$ and $\xi=0.05$.

\section{B. Extension of Tsypkin's Method to Mixed-Signal Systems}

We will presently show how Tsypkin's criterion may be used to determine the oscillation frequencies of systems $S^{1}$ or $S^{2}$. Let us first consider system $S^{1}$ of Fig. 3. There are two reasons why applying Tsypkin's method "as is" to this system is not very practical for the following reasons:

- the spectrum folding due to the presence of the sampled system, the Fourier series representation of the relay's input involves a double sum;

- the delay $\tau$ between the relay's switching moment and the next sampling moment is a priori unknown.

In order to apply Tsypkin's method to $S^{1}$ or $S^{2}$, they must first be transformed into a particular-or "canonical"-form by subjecting them to a series of elementary operations. After this preliminary step, we will show how Tsypkin's criterion may be extended to the canonical system to determine its oscillation frequencies.

1) Canonical Form of the System: Let us transform system $S^{1}$ so that the discrete part consists only of a switch and of a zeroth-order hold. This can be done by replacing the unit discrete-time delays of filter $H(z)$ by unit continuous-time delays and by placing the resulting filter $H\left(\exp \left(s T_{s}\right)\right)$ after the zeroth-order hold (Fig. 6). This transformation is in no way approximate because $H\left(\exp \left(s T_{s}\right)\right)$ only "sees" sampled-and-held signals. Because of this equivalence between the original and the transformed system, it is clear that neither the output of the comparator nor its input have changed.

The discrete part of the system now consists only of a switch and of a zeroth-order hold, and the transformation can be completed by swapping the discrete-time block and the comparator. This transformation leaves the output of the continuous block unchanged because both operations (sampling and taking the sign) commute as long as the sampling moments are equivalent (Fig. 7). It is then equivalent to study the oscillations of the original system $S^{1}$ and those of its canonical form $S^{0}$. System $S^{0}$ is represented in Fig. 8.

In order to transform $S^{2}$ into its canonical form, only one transformation step is needed: the discrete-time delays are changed into continuous-time delays, and the resulting filter is placed between $G(s)$ and the sampling stage. This leaves the output of $G(s)$ unchanged and, thus, $S^{2}$ is transformed into its equivalent canonical form $S^{0}$.

For example, to obtain the canonical form of the pulse-delivering system of Fig. 2, one needs to follow the transformation steps shown in Fig. 9. 

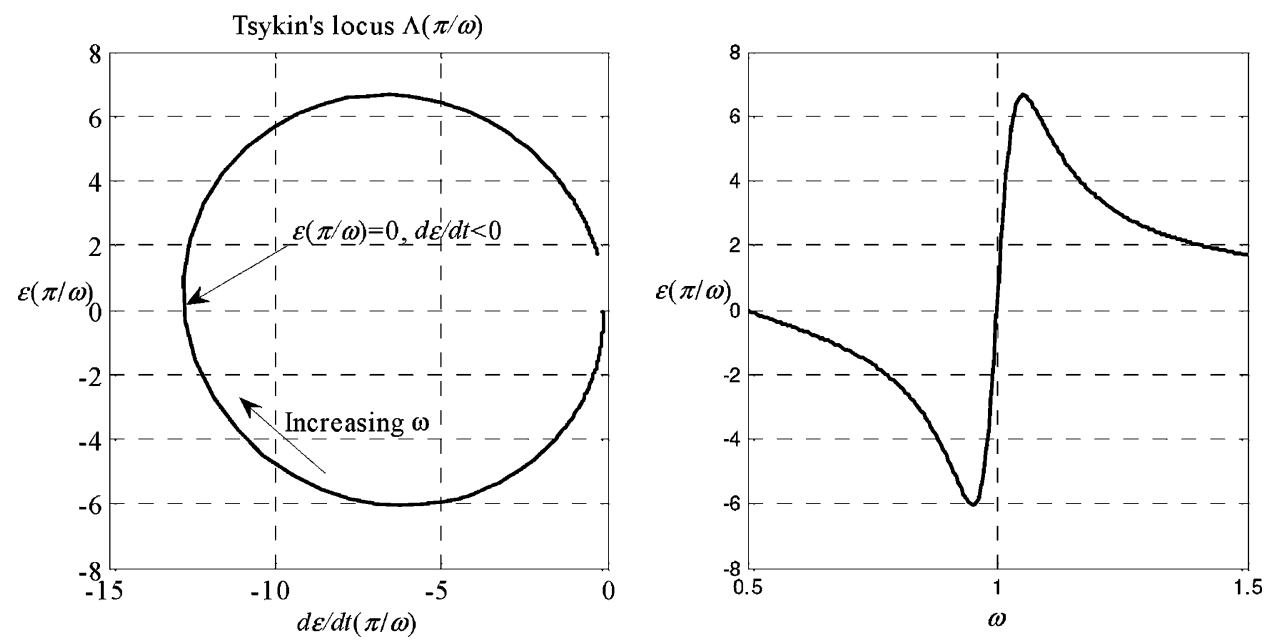

Fig. 5. Typical Tsypkin's locus $\Lambda(\pi / \omega)$ for the system of Fig. 1 (left). The value of the oscillation period is given by the intersection with the real axis. $\varepsilon(\pi / \omega)$ is represented on the right.

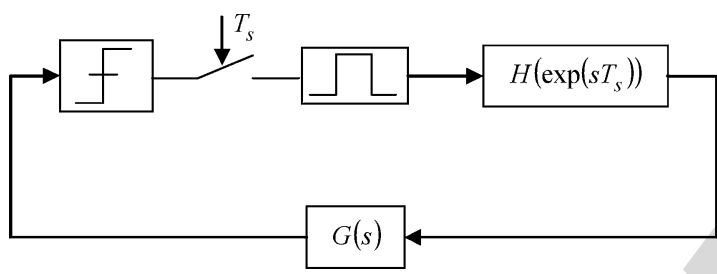

Fig. 6. System $S^{1}$ is transformed into an equivalent system, in which the discrete delays have been replaced by continuous ones.

2) Application of Tsypkin's Criterion to a Canonical System in the Case of Simple Oscillations: Let us first consider the case of simple oscillations of the canonical system $S^{0}$, i.e., the case when the output of the comparator is a periodic square signal with amplitude $M$. Due to the fact that the relay cannot switch between samples because of the presence of the zeroth-order hold, oscillations with period $T_{0}$ are possible if and only if $T_{0}$ is an even multiple of sampling period $T_{s}$

$$
\exists n \in \mathrm{N} / T_{0}=2 n T_{s} .
$$

Since the relay's input $\varepsilon(t)$ is equal to $f(t)$ at any sampling moment, the switching conditions can be written as

$$
\left\{\begin{array}{l}
\varepsilon\left(\frac{T_{0}}{2}-T_{s}\right)=f\left(\frac{T_{0}}{2}-T_{s}\right)>0 \\
\varepsilon\left(\frac{T_{0}}{2}\right)=f\left(\frac{T_{0}}{2}\right)<0 .
\end{array}\right.
$$

Now, following Tsypkin's method, the origin of time is taken on a rising edge of the comparator. $s(t)$ then has the following Fourier series development:

$$
s(t)=\frac{2 j M}{\pi} \sum_{k \text { odd }} \frac{\exp \left(j k \frac{2 \pi}{T_{0}} t\right)}{k} .
$$

In the frequency domain, this is equivalent to

$$
\tilde{s}(\omega)=4 j M \sum_{k \text { odd }} \frac{\delta\left(\omega-k \frac{2 \pi}{T_{0}}\right)}{k} .
$$

The output of the continuous block may then be written as

$$
\tilde{f}(\omega)=4 j M \sum_{k \text { odd }} F\left(k \frac{2 \pi}{T_{0}}\right) \frac{\delta\left(\omega-k \frac{2 \pi}{T_{0}}\right)}{k} .
$$

In the time domain, (4) can be rewritten as

$$
\left\{\begin{array}{c}
\varepsilon^{-}=\varepsilon\left(\frac{T_{0}}{2}-T_{s}\right)=-\frac{2 j M}{\pi} \sum_{k \text { odd }} \frac{1}{k} F\left(k \frac{\pi}{n T_{s}}\right) \\
\exp \left(-j \frac{k \pi}{n}\right)>0 \\
\varepsilon^{+}=\varepsilon\left(\frac{T_{0}}{2}\right)=-\frac{2 j M}{\pi} \sum_{k \text { odd }} \frac{1}{k} F\left(k \frac{\pi}{n T_{s}}\right)<0 .
\end{array}\right.
$$

Verifying (8) now only requires computing the values of integer $n$ that meet the switching conditions. As opposed to the continuous-time case, the oscillation frequency can always be exactly determined.

Implementation of (8) is fairly straightforward and leads to very fast calculations. One must keep in mind that (8) may have to be adapted to take into account possible poles of $F(s)$ in $s=0$. In this situation, an arbitrary offset, which depends on the initial state of $S^{0}$, must be added to the oscillation criterion. (8) then becomes

$$
\min \left(\varepsilon\left(\frac{T_{0}}{2}-T_{s}\right), \varepsilon\left(T_{0}\right)\right)>\max \left(\varepsilon\left(\frac{T_{0}}{2}\right), \varepsilon\left(T_{0}-T_{s}\right)\right) .
$$

For example, let us consider the system of Fig. 2, with $\omega_{0}=$ $1, \xi=0.05$, and $T_{s}=0.4$. The extended Tsypkin's locus

$$
\Lambda^{\prime}\left(\frac{\pi}{\omega}\right)=\varepsilon_{+}\left(\frac{\pi}{\omega}\right)+j \varepsilon_{-}\left(\frac{\pi}{\omega}\right)
$$

is shown in Fig. 10. This figure shows that oscillations may take place in the system with period $T_{0}=16 T_{s}$ or with period $T_{0}=$ $48 T_{s}$.

Finally, one should check for supplementary zero-crossings of $\varepsilon(t)$ between 0 and $T_{0}$ : if there are none, then the predicted oscillations are correct and may occur in the system. For the chosen set of parameters $\omega_{0}, \xi$, and $T_{s}$, the time response of the system is shown in Fig. 11, for $T_{0}=16 T_{s}$. For $T_{0}=48 T_{s}$, several supplementary zero-crossings can be found. 


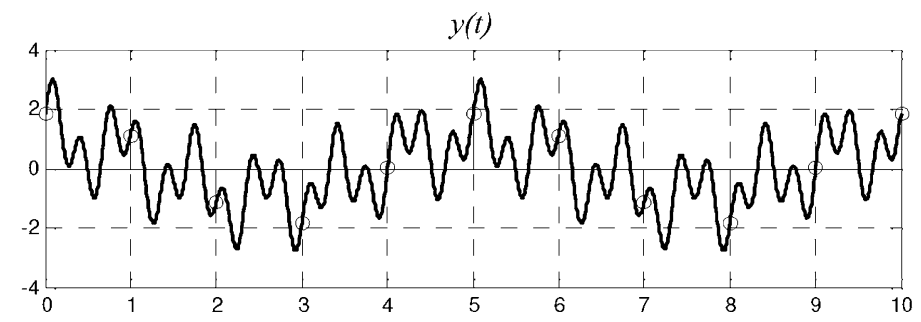

(a)

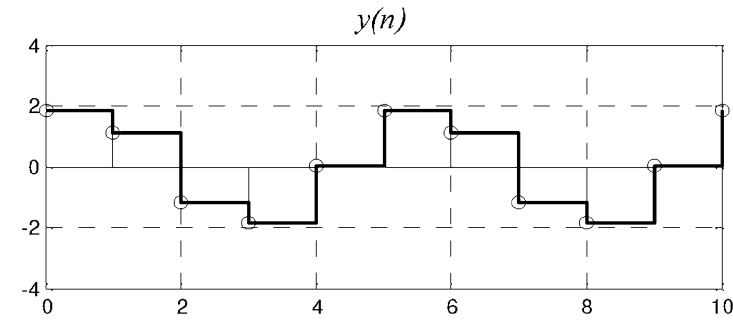

(b)

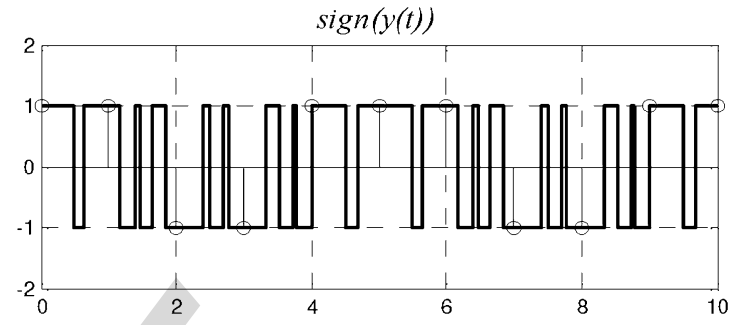

(c)

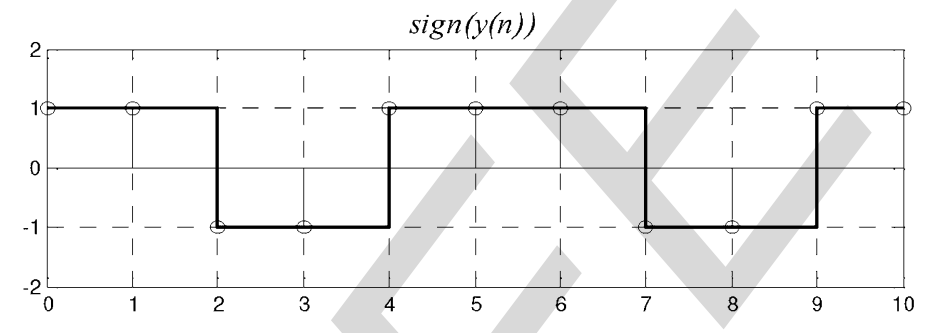

(d)

Fig. 7. (a) Original signal. The result (d) is identical whether the original signal is (b) first sampled (c) or not, as long as the sampling moments (circles) are the same.

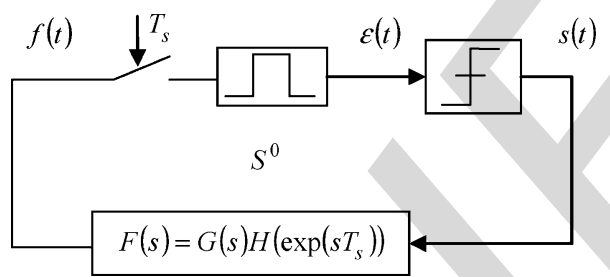

Fig. 8. Canonical form $S^{0}$ of systems $S^{1}$ and $S^{2}$.

Let us now consider the case when the portions of the extended Tsypkin's locus that belong to the upper left quadrant (if any) do not contain any point that verifies $T_{0}=2 n T_{s}$ (Fig. 12). Let us also suppose that these portions correspond values of $T_{0}$ comprised between $2 n T_{s}$ and $2(n+1) T_{s}$. From continuous-time theory, it is intuitive that the system should oscillate with a period belonging to this range. Nevertheless, due to the width-quantization effect of the sampling-and-holding stage, this is not possible stricto sensu and results in complex oscillations of the system, which we will discuss next.

3) Application of Tsypkin's Criterion to a Canonical System in the Case of Complex Oscillations: There are many situations in which the relay's output is no longer a simple square-wave with period $2 n T_{s}$. This is in fact much more common in hybrid systems than in continuous ones. For example, consider the case of the system shown in Fig. 1. In the absence of a samplingand-holding stage, an arbitrarily small change in the spring's stiffness results in a small change in the oscillation frequency, i.e., in the width of the square wave. This is no longer possible in the case of the system of Fig. 2, because the width of the square wave has to be an integer multiple of $T_{s}$ and it is, therefore, quantized. However, the relay's output can still be a periodic signal. Let us call $T_{0}$ its period and $q$ the number of times the comparator switches to the high position during period $T_{0}$. We call "sub-period" the time between any two successive rising edges - there are $q$ sub-periods in period $T_{0}$. Each sub-period is divided in two intervals - there are $2 q$ such intervals in period $T_{0}$ (Fig. 13). Symbols $W_{k}$ and $V_{l}$ designate the number of samples (or width) of the $k$ th sub-period and the width of the $l$ th interval, respectively.

We call "mean period" the quantity

$$
T_{m}=\frac{T_{0}}{q} \text {. }
$$

It is the mean period i.e., the average value of the time between two rising edges, that most period-meters measure [20]. Since $T_{0}$ is necessarily an integer multiple of the sampling period, it follows that:

$$
\frac{T_{m}}{T_{s}} \in \mathrm{Q}
$$

and

$$
\exists r \in \mathrm{N} / r \leq \frac{T_{m}}{T_{s}}<r+1 .
$$

We notice that small changes in any of the system's parameters induce changes in the value of $T_{m}$. For example, the value of the measured period versus the system's stiffness $k$ has been 


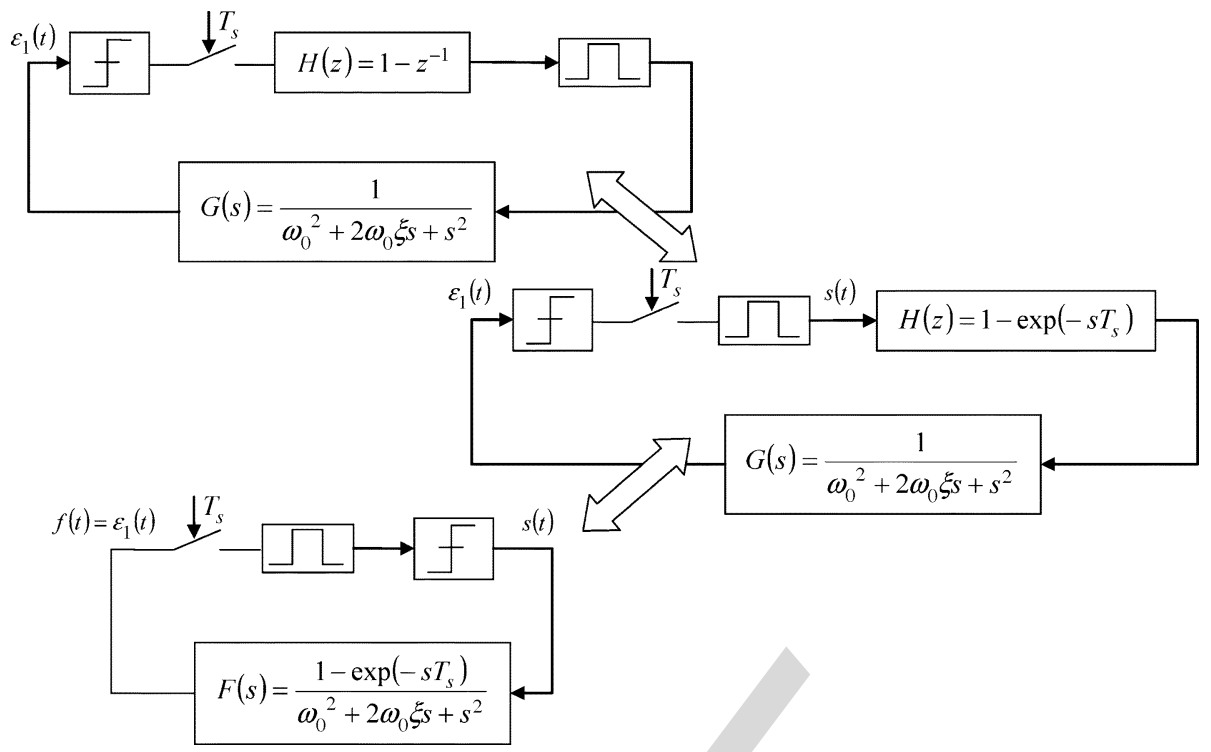

Fig. 9. System of Fig. 2 is transformed in its canonical form in three steps.

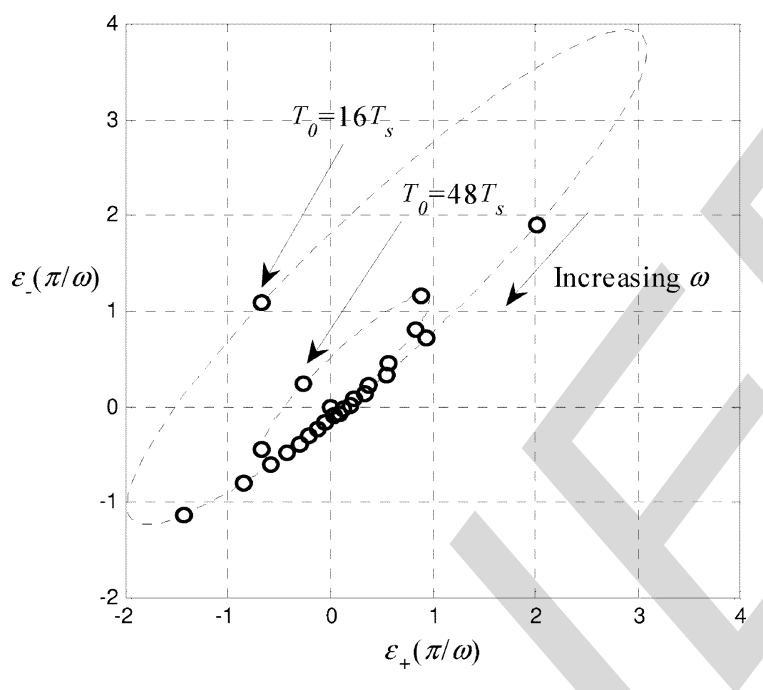

Fig. 10. Extended Tsypkin's locus $\Lambda^{\prime}(\pi / \omega)$ for the system of Fig. 2. The circles correspond to values of $T_{0}$ that are even multiples of the sampling period. An oscillation with period $T_{0}=2 n T_{s}$ may occur in the mixed-signal system iff the corresponding circle lies in the upper left quadrant [from (8)].

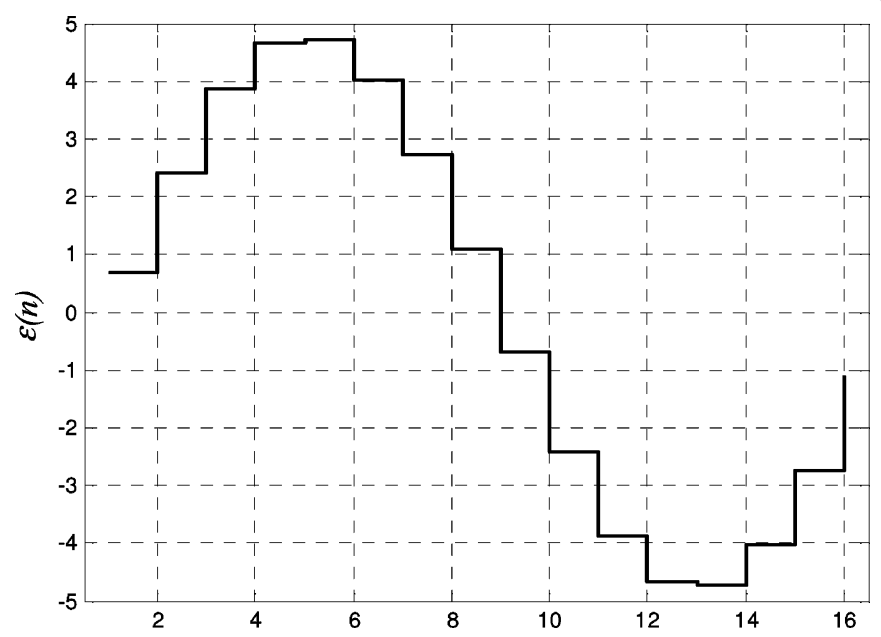

Fig. 11. $\varepsilon(t)$ for $T_{0}=16 T_{s}$.
Fig. 12. The only value of $T_{0}=2 n T_{s}$ which belongs to the upper-left quadrant is $T_{0}=52 T_{s}$. However, this oscillation period is not possible (because of the existence of several supplementary zero-crossings). Nevertheless, the curve suggests that a limit cycle may exist between $T_{0}=16 T_{s}$ and $T_{0}=18 T_{s}$.

plotted in Fig. 14, for the continuous system of Fig. 1 and for the hybrid system of Fig. 2 with $T_{s}=0.4 \mathrm{~s}$ and

$$
G(s)=\frac{1}{k+0.1 s+s^{2}} .
$$

These changes in the mean period correspond to variations in the number of sub-periods $(q)$ and in the (quantized) widths of these sub-periods. Hence, in an averaged sense, the apparent period of the system is not necessarily an even integer multiple of the sampling period. The remainder of this paper is devoted to the common case of systems for which the width of each sub-period only takes two values, i.e., $W_{k}=r$ or $W_{k}=r+1$, and $r$ is defined in (12).

In this case, we consider only three possible sub-periods, which are shown in Fig. 15. We now restate Tsypkin's criterion in the case of complex oscillations of hybrid systems. If the 


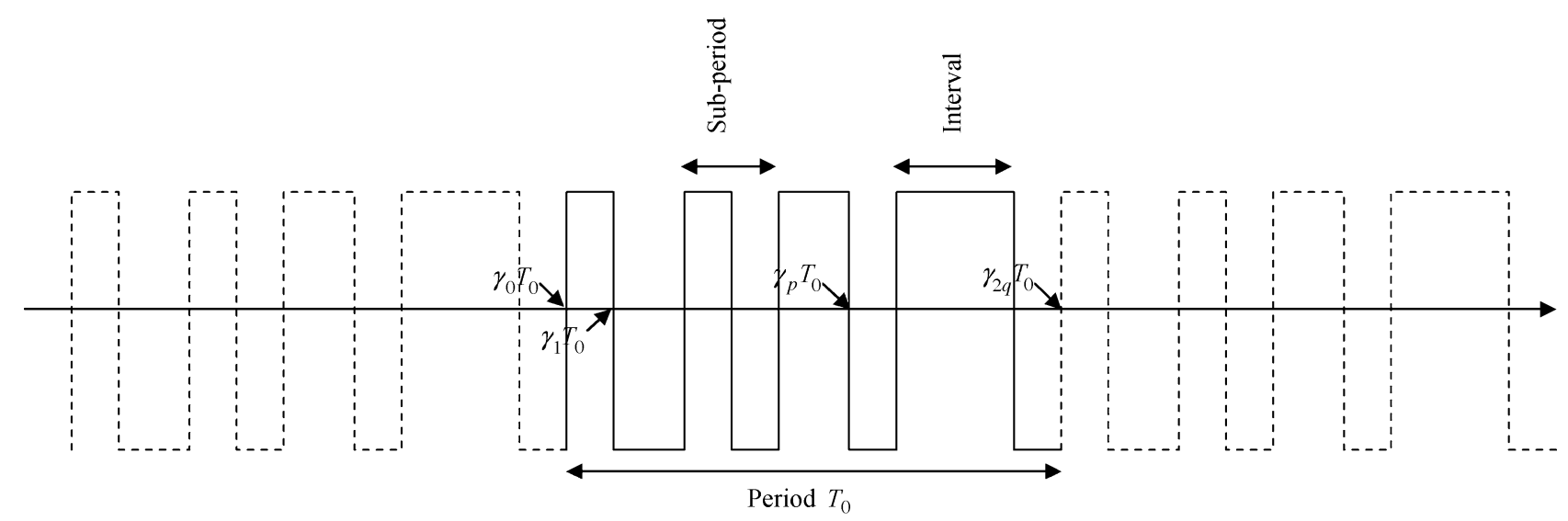

Fig. 13. Complex oscillations with $q=4$ sub-periods.

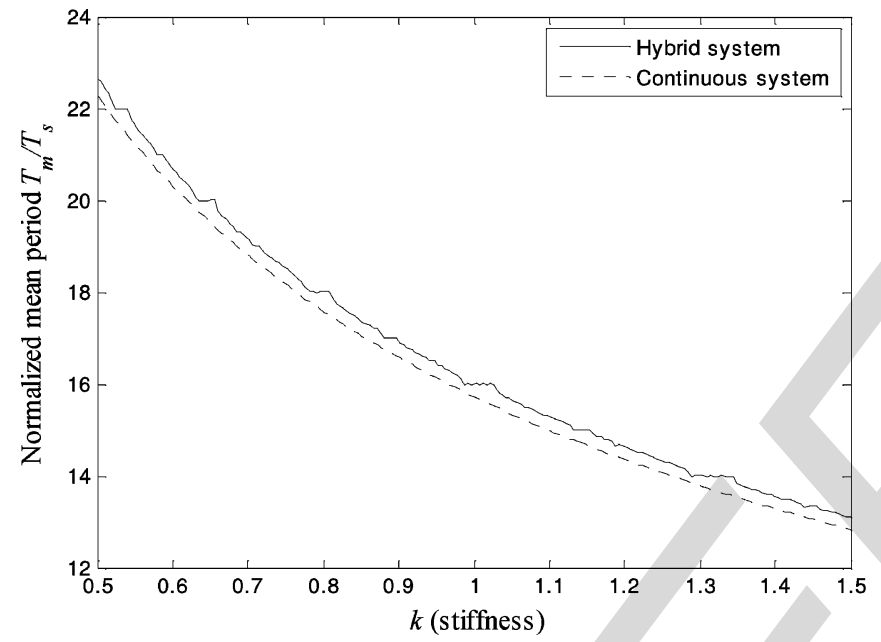

Fig. 14. Changes in the system's stiffness induce changes in the period of the continuous system as well as in the mean period of the hybrid system. Noninteger and odd values of $T_{m} / T_{s}$ denote the existence of complex oscillations. The hybrid system's response is similar to the devil's staircases appearing in $\Sigma \Delta$ modulation [16].

relay's output $s(t)$ is periodic, it can be expressed as a Fourier series

$$
\tilde{s}(\omega)=2 \pi \sum_{k} \alpha_{k} \delta\left(\omega-k \frac{2 \pi}{T_{0}}\right)
$$

Taking the origin of time on a rising edge yields

$$
\left\{\begin{array}{l}
\alpha_{0}=-M\left[1+2 \sum_{p=1}^{2 q-1}(-1)^{p} \gamma_{p}\right] \\
\alpha_{k}=\frac{M}{\pi j k}\left[1-\sum_{p=1}^{2 q-1}(-1)^{p} \exp \left(-2 \pi j k \gamma_{p}\right)\right], \quad \text { if } k \neq 0
\end{array}\right.
$$

and $\left.\gamma_{p} \in\right] 0.1[$. The same notation as in [18] is used here, so that $\gamma_{p}$ designates the $(p+1)$ th switching moment (Fig. 13). The $\gamma_{p}$ are all necessarily rational numbers. An odd subscript denotes a falling edge, while an even subscript denotes a rising edge.

Oscillations are possible only if the switching conditions (13) are verified for $\left(\gamma_{p}\right)_{1 \leq p<2 q}$

$$
\left\{\begin{array}{l}
\varepsilon_{p}^{-}=\varepsilon\left(\gamma_{p} T_{0}-T_{s}\right)= \\
(-1)^{p} \sum_{k} \alpha_{k} F\left(k \frac{2 \pi}{T_{0}}\right) \exp \left(j k 2 \pi\left(\gamma_{p}-\frac{T_{s}}{T_{0}}\right)\right)<0 \\
\varepsilon_{p}^{+}=\varepsilon\left(\gamma_{p} T_{0}\right)= \\
(-1)^{p} \sum_{k} \alpha_{k} F\left(k \frac{2 \pi}{T_{0}}\right) \exp \left(j 2 \pi k \gamma_{p}\right)>0 .
\end{array}\right.
$$

As stated in Section 2-B-2, this criterion may be adapted if transfer function $F(s)$ has a pole in $s=0$

$$
\min _{0 \leq n \leq q}\left(\left\{\varepsilon_{2 n+1}^{-}\right\} \cup\left\{\varepsilon_{2 n}^{+}\right\}\right)>\max _{0 \leq n \leq q}\left(\left\{\varepsilon_{2 n}^{-}\right\} \cup\left\{\varepsilon_{2 n+1}^{+}\right\}\right) \text {. }
$$

One must note that (13) and (14) are valid even if there are more than three possible sub-periods. In the most general case, as in the case of complex oscillations of continuous systems, there is no simple way of determining the shape of the complex oscillations (the $\gamma_{p}$ ): one may try to maximize a scalar product between a trial shape and the corresponding system output. Nevertheless this is rather unpractical because of the relay's nonlinearity. One may also view this problem as a frequency-modulation or pulse-width modulation issue. In the case there are only three possibilities for each of the sub-periods, Tsypkin's method can be taken one step further and one may try to determine the shape of the complex oscillations in a relatively simple way.

4) Practical Determination of Oscillation Shape: As it has already been stated, the value of $r$ may be deduced from the curves obtained when checking for simple oscillations. The most direct method for building the complex oscillations then consists in trying, for a given $q$ and a given $r$, all possible combinations of sub-periods. Since there are only three possibilities for each sub-period (Fig. 15) and because of the $T_{0}$-periodicity of the pattern, finding all combinations is equivalent to solving the so-called "necklace problem": finding all the different necklaces that can be made with $q$ beads of $n$ different colours. The specificity of the problem lies in the topological invariance of the necklace through any rotation [21]. Here, each possible sub-period (Fig. 15) corresponds to a colour and the periodicity of the complex oscillations corresponds to the rotation invariance of the necklace. However, solving the necklace problem with $q$ beads of three different colours yields about $3^{q} / q$ different combinations [22]. Testing all combinations becomes very lengthy as $q$ grows and limits the applicability of this 

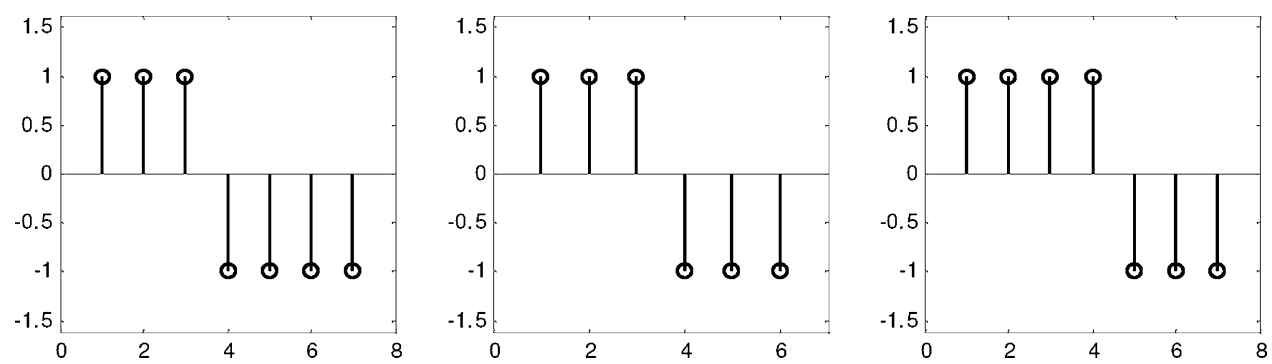

Fig. 15. For a given value of $r$, only three possible sub-periods are considered. Here, $r=6$. The resulting mean period $T_{m}$ lies between $6 T_{S}$ and $7 T_{s}$.
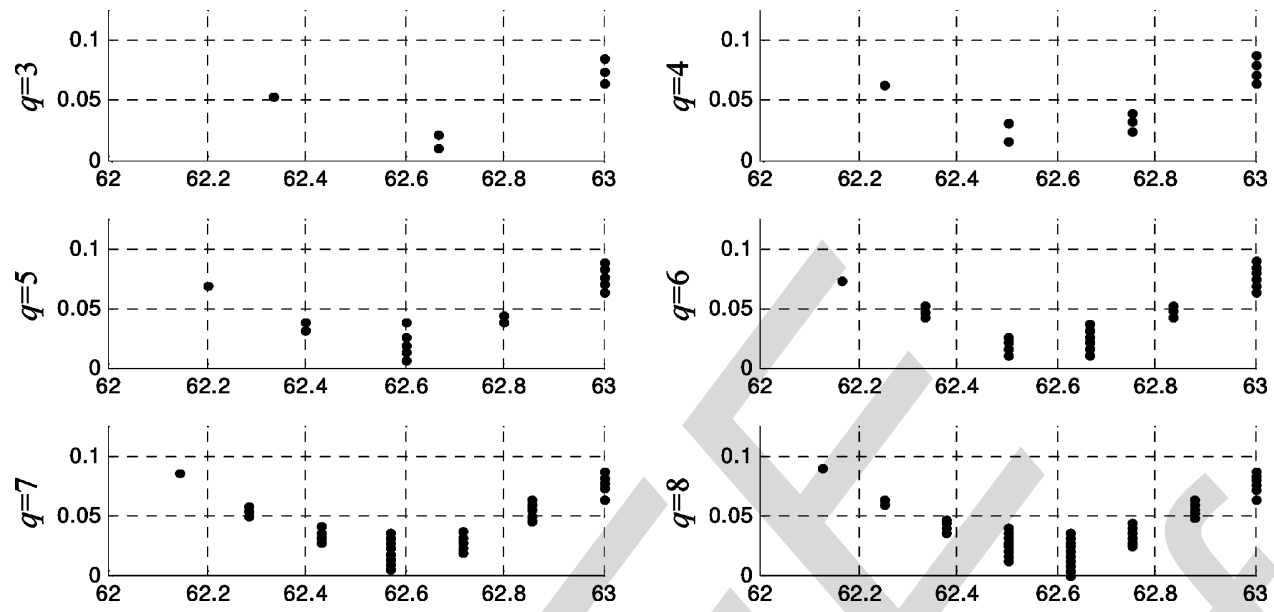

Fig. 16. Costs obtained for all necklace combinations as a function of the mean period. Here, the solution is obtained for $q=8$ and $T_{m}^{s o l}=62+5 / 8$. For each value of $q \leq 8$, the cost is minimized for a combination corresponding to the value of $T_{m}$ closest to $T_{m}^{s o l}$. For example, there are three possible values of the mean period for $q=3$ (upper left figure) and the cost function is minimal for a necklace with mean period $T_{m}=62+2 / 3$.

method to $q \leq 12$ on a standard PC ( $2 \mathrm{GHz}$ CPU, 1 Go RAM). Other methods must then be used to speed up the process of characterizing the complex oscillations of the system.

Let us suppose we are searching for the form of the quantized complex oscillations of a given system. As stated in the previous paragraph, the most straightforward manner consists in trying for each $q$ all combinations of widths. One may give this problem a more formal definition by introducing for each combination a cost as

$$
\text { Cost }=\frac{|L|}{q}, L=\left\{p / \text { criterion is not met in } \gamma_{p}\right\}
$$

and by minimizing it. In (15), $|L|$ stands for the cardinality of set $L$. This cost function may vary between 0 and 1,0 corresponding to possible oscillations of the system. Typical results are shown in Fig. 16.

As it can be seen in Fig. 16, one may try at the $q$ th step to use the information contained in the previous steps and focus on necklaces of a given density (mean period) in order to accelerate the convergence of the process: necklace combinations with densities too different from that of the minimum of the previous step are not tested. Depending on the value of the mean period, this can greatly reduce the computational costs. This method can also be used if only an estimation of $T_{m}$ is needed.

Another intuitive method for reducing the number of trials is, for a given mean period $T_{m}$ and a given $q$, to concentrate on the necklaces yielding the periodic patterns that are most similar to a square wave of period $T_{m}$. This idea is based on the assumption that the system has as "periodic" a behaviour as possible and that the comparator's output is, on the whole, as "square" as possible. A systematic solution to this problem [16], [17] is to use Euclid's algorithm. The same results may also be obtained by using the more straightforward approach described in the next paragraph.

For a given value of $T_{m}$ and of $q$, let $p$ be the integer such that

$$
\frac{T_{m}}{T_{s}}=r+\frac{p}{q}, p \leq q .
$$

Let us suppose, to simplify notations, that $r$ is even. If $r$ is odd, one may write

$$
\frac{T_{m}}{T_{s}}=(r+1)-\frac{p}{q}
$$

and the following method may also be applied. If $p$ and $q$ are co-prime, the sequences of intervals $\left(V_{1}, V_{2}, \ldots, V_{2 q}\right)$ that most resemble a square wave of period $T_{m}$ are

$$
V_{k, k \in\{1, \ldots, 2 q\}}=\frac{r}{2}+\left\lceil\frac{k}{2} \frac{p}{q}+\frac{1}{2}\right\rceil-\left\lceil\frac{(k-1)}{2} \frac{p}{q}+\frac{1}{2}\right\rceil
$$

or by

$$
V_{k, k \in\{1, \ldots, 2 q\}}=\frac{r}{2}+\left\lfloor\frac{k}{2} \frac{p}{q}+\frac{1}{2}\right\rfloor-\left\lfloor\frac{(k-1)}{2} \frac{p}{q}+\frac{1}{2}\right\rfloor .
$$

In (16) and (17), $\lfloor x\rfloor$ denotes the floor function and $\lceil x\rceil$ the ceiling function. If $p$ is odd, these equations yield two different sequences. However, (16) and (17) yield the same result if $p$ is even. It must be noted that the periodic patterns generated with (16) and (17) maximize the circular correlation with a square wave of period $T_{m}$. For example, for $\left(T_{m} / T_{s}\right)=r+(2 / 3)$, (16) or (17) yields 


$$
\left(V_{k}\right)_{k \in\{1, \ldots, 6\}}=\frac{r}{2}+(0,1,0,0,1,0) .
$$

For $\left(T_{m} / T_{s}\right)=r+(1 / 3),(16)$ gives

$$
\left(V_{k}\right)_{k \in\{1, \ldots, 6\}}=\frac{r}{2}+(0,0,0,1,0,0)
$$

and (17) gives

$$
\left(V_{k}\right)_{k \in\{1, \ldots, 6\}}=\frac{r}{2}+(0,0,1,0,0,0) .
$$

These two results are equivalent through a one interval shift, but no sub-period shift can transform one into the other.

Let us now consider the case when

$$
\frac{T_{m}}{T_{s}}=r+\frac{P}{Q}
$$

and $P$ and $Q$ are not co-prime: in that case, Euclid's algorithm no longer applies. Let us then call $N$ their greatest common divisor, such that

$$
\left\{\begin{array}{l}
P=N p \\
Q=N q
\end{array}\right.
$$

One can build some sequences of $2 Q$ intervals that maximize the correlation with a square wave of period $T_{m}$ in the following way. First, we use (16) and (17) to build the $2 q$-long sequence(s) corresponding to $T_{m}$. If $p$ is even, the $2 Q$-long sequence is obtained by repeating the elementary sequence $N$ times: it is therefore unnecessary to test this possibility since it will lead to the same results as those of the elementary sequence. If $p$ is odd, the sequence is built like a necklace of length $N$ with beads of two colours, corresponding to the two elementary sequences obtained with (16) and (17). The sequences built in such a way also maximize the circular correlation with a square wave of period $T_{m}$. For example, for $\left(T_{m} / T_{s}\right)=r+(2 / 6)$, three possibilities need to be tested

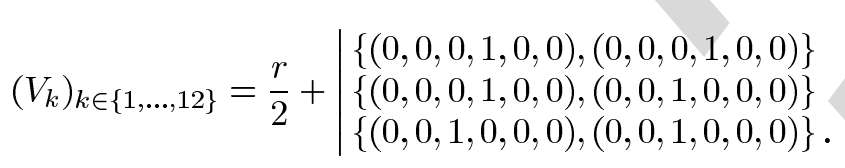

For $\left(T_{m} / T_{s}\right)=r+(3 / 9)$, four possibilities need to be tested

$$
\begin{aligned}
\left(V_{k}\right)_{k \in\{1, \ldots, 18\}}=\frac{r}{2} & \\
+ & \begin{array}{l}
\{(0,0,0,1,0,0),(0,0,0,1,0,0),(0,0,0,1,0,0)\} \\
\{0,0,0,1,0,0),(0,0,0,1,0,0),(0,0,1,0,0,0)\} \\
\{0,0,0,1,0,0),(0,0,1,0,0,0),(0,0,1,0,0,0)\} \\
\{(0,0,1,0,0,0),(0,0,1,0,0,0),(0,0,1,0,0,0)\} .
\end{array}
\end{aligned}
$$

We refer to this method as the "reduced necklace" method. This method has a much lower computational cost than the "full necklace" method. Moreover, it is possible to test for very long limit cycles (up to $q=200$ on a standard PC) when using the reduced necklace method. Finally, in all the cases on which the reduced necklace was tested, the "full necklace" did not yield any supplementary solution. Although this observation consti-

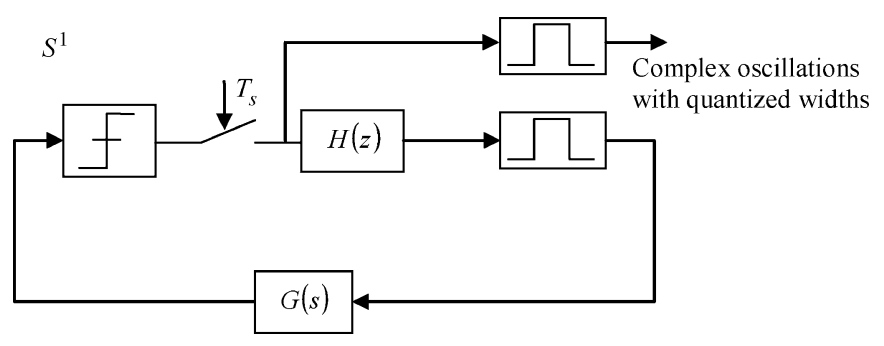

Fig. 17. Quantized complex oscillations determined for $S^{0}$ (Fig. 8) can be observed in $S^{1}$ [Fig. 3(a)] by adding a zeroth-order hold directly after the sampling stage.

tutes no formal proof, it points at the relevance of the reduced necklace method.

Finally, an alternate method for reducing the computational costs is simply to evaluate the time response of $F(s)$ to the complex oscillations as a finite sum over its poles, as in Hamel's method [19], rather than as an infinite Fourier series.

\section{Application And Results}

\section{A. Non-Canonical Case}

It has been shown in Section II how the characteristics-mean period, sub-periods - of a canonical system may be calculated. Let us consider the case of a mixed-signal nonlinear system that is not already in the canonical form, say the system of Fig. 2. The proposed method still yields the correct value of the relay's input at the sampling moments and the correct value of the mean period of the system, which are unchanged by the transformation to the noncanonical case. However, the signal at the relay's output does not necessarily correspond to the quantized complex oscillations we determined in Section II. In the case of system $S^{1}$, these can be observed by simply adding a sampling-and-holding stage at the output of the relay, as shown in Fig. 17. In the case of system $S^{2}$, the complex oscillations with quantized widths can be observed directly at the comparator's output.

\section{B. Results}

To illustrate the proposed approach, let us first consider a system composed of a continuous second-order system

$$
G(s)=\frac{1}{s^{2}+0.1 s+1}
$$

an ideal comparator, and a discrete-time integrator

$$
H(z)=\frac{z}{1-z}
$$

with sampling period $T_{s}=0.1 \mathrm{~s}$. In order to determine the oscillation frequency of this system, the following three methods are used:

- a classical Tsypkin analysis, in which the effect of sampling is neglected;

- a mixed-signal simulation, performed with Matlab/ Simulink, in which the value of the mean period is obtained by averaging the time between two rising edges at the comparator's output; 
- an extended Tsypkin analysis, as described in the previous parts, using criterion (14) because of the presence of the integrator.

In a classical Tsypkin analysis of the system, the influence of sampling is neglected and Tsypkin's criterion is applied to the continuous transfer function

$$
F(s)=\frac{\exp \left(s T_{s}\right)}{1-\exp \left(s T_{s}\right)} \frac{1}{s^{2}+0.1 s+1} .
$$

The calculated oscillations are found to have approximately pe$\operatorname{riod} T_{0}=6.268 \mathrm{~s}$.

This result is inaccurate because both the simulation and the extended Tsypkin's criterion (14) produce $T_{m}=6.28 \mathrm{~s}$. However, the accuracy of the simulation depends on the number of points taken to obtain the average value of the "period" of the oscillations. The result is exact only when a multiple of 5 is chosen because the mean period of the comparator's output is actually

$$
T_{m}=\left(62+\frac{4}{5}\right) T_{s}
$$

as obtained by the calculation of the extended Tsypkin's criterion.

If we slightly change the parameters of the continuous system to

$$
G(s)=\frac{1}{s^{2}+0.1 s+1.01}
$$

the extended Tsypkin's criterion (14) predicts different oscillation possibilities, depending on the following initial conditions.

- $T_{m}=62.5 T_{s}=(62+(2 / 4)) T_{s}$ with

$$
\left(V_{k}\right)_{k \in\{1, \ldots, 8\}}=31+(0,0,1,0,0,1,0,0) .
$$

- $T_{m}=62.5 T_{s}=(62+(4 / 8)) T_{s}$ with

$\left(V_{k}\right)_{k \in\{1, \ldots, 16\}}=31+(0,0,1,0,0,0,1,0,0,1,0,0,0,1,0,0)$.

- $T_{m}\left(=62.55 T_{s}\right)=(62+(5 / 11)) T_{s}$ with

$\left(V_{k}\right)_{k \in\{1, \ldots, 22\}}=31$

$+(0,0,1,0,0,0,1,0,0,0,1,0,0,0,0,1,0,0,0,1,0,0)$.

- $T_{m}\left(=62.53 T_{s}\right)=(62+(8 / 15)) T_{s}$ with

$\left(V_{k}\right)_{k \in\{1, \ldots, 30\}}=31+(0,1,0,0,0,1,0,0,0,1,0$,

$$
0,0,1,0,0,1,0,0,0,1,0,0,0,1,0,0,0,1,0) \text {. }
$$

The reduced necklace method was used to determine the shape of these oscillations. We have not checked for solutions with $q>$ 12 , except for co-prime $p$ and $q$, for which there did not seem to be other possible oscillations for $q \leq 32$. The full necklace method was used in order to find other possible solutions, but none were found.

These values of $T_{m}$ may be compared with the output of Tsypkin's method for continuous systems, which yields a periodic oscillation with $T_{0}=62.68 T_{s}$, or with those obtained with a mixed-signal simulation. In the latter case, the complete range of possible initial conditions must be tried if all possible oscillations are to be found. Added to the relative inaccuracy of the computed value of the mean period, it makes the simulation approach rather unpractical and, in our point of view, stresses the interest of our own method.

\section{CONCLUSION}

In this paper, we have shown how Tsypkin's approach for determining the oscillation frequencies of continuous nonlinear systems could be extended to a class of mixed-signal nonlinear systems, consisting of one possibly nonideal relay, one discrete-time system with a zeroth-order hold, and one continuous system. Starting from a typical configuration, we showed how to convert these systems into an equivalent canonical form to which Tsypkin's approach can be applied. Then, we considered the case of simple oscillations of the system for which it was shown that the period is necessarily an even multiple of the sampling period. Two oscillation criteria were established, depending on whether the equivalent continuous transfer function has a pole in zero or not. The case of complex oscillations of the system was addressed in a similar fashion and, because of the quantization effects induced by the discrete-time stage, Tsypkin's approach could then be taken one step further: we showed how, using combinatory analysis and a simplified version of Euclid's algorithm, the shape of the complex oscillations could be determined in many situations. We also illustrated this approach and proposed several methods for improving its implementation.

These theoretical results can be used for the design and the analysis of resonant MEMS. Promising developments include the study of the impulse-delivering system [10] of Fig. 2 or the use of the shape of the quantized complex oscillations to determine the parameters of simple systems [15].

\section{ACKNOWLEDGMENT}

The authors would like to thank the referees for their helpful comments and the associate editor for her patience.

\section{REFERENCES}

[1] M. Bao and W. Wang, "Future of microelectro-mechanical systems," Sensors Actuat., vol. A56, no. 1-2, pp. 135-141, Aug. 1996.

[2] S. Sung, J. G. Lee, T. Kang, and J. W. Song, "Development of a tunable resonant accelerometer with self-sustained oscillation loop," in Proc. IEEE National Conf. Aerosp. Electron., Dayton, OH, Oct. 2000, pp. 354-361.

[3] K. Browns, W. Allegretto, F. E. Vermeulen, and A. M. Robinson, "Simple resonating microstructures for gas pressure measurement," $J$. Micromech. Microeng., vol. 12, pp. 204-210, May 2002.

[4] N. Yazdi, F. Ayazi, and K. Najafi, "Micromachined inertial sensors," Proc. IEEE, vol. 86, no. 8, pp. 1640-1659, Aug. 1998.

[5] C. Nguyen and R. Howe, "An integrated CMOS micromechanical resonator high- $Q$ oscillator," IEEE J. Solid-State Circuits, vol. 34, no. 4, pp. 440-455, Apr. 1999.

[6] L. Lin, R. T. Howe, and A. P. Pisano, "Microelectromechanical filters for signal processing," IEEE J. Microelectromech. Syst., vol. 7, no. 3, pp. 286-294, Sep. 1998

[7] T. Corman, K. Noren, P. Enoksson, J. Melin, and G. Stemme, "Burst technology with feedback-loop control for capacitive detection and electrostatic excitation of resonant silicon sensors," IEEE Trans. Electron. Devices, vol. 47, no. 11, pp. 2228-2235, Jun. 2000.

[8] S. Sung, J. G. Lee, B. Lee, and T. Kang, "Design and performance test of an oscillation loop for a MEMS resonant accelerometer," J. Micromech. Microeng., vol. 13, no. 2, pp. 246-253, Mar. 2003.

[9] S. Pavan and Y. P. Tsividis, "An analytical solution for a class of oscillators, and its application to filter tuning," IEEE Trans. Circuits Syst. I, Fundam. Theory Appl., vol. 45, no. 5, pp. 547-555, May 1998. 
[10] E. Colinet, J. Juillard, S. Guessab, and R. Kielbasa, "Actuation of resonant MEMS using short pulsed forces," Sensors Actuat., vol. A115, no. 1, pp. 118-125, Sep. 2004.

[11] O. P. McNamara and D. P. Atherton, "Limit cycles in nonlinear sampled data systems," in Proc. 9th Triennial World Congress IFAC, 1985, vol. 1, pp. 507-512.

[12] A. Goucem and D. P. Atherton, "Limit cycles in nonlinear discrete systems," in Proc. 25th IEEE Conf. Decision and Control, Dec. 1986, vol. 1, pp. 573-577.

[13] C. P. Lewis, "Prediction of limit cycle conditions in nonlinear sampled data systems," Trans. Inst. Measur. Contr., vol. 1, no. 4, pp. 199-203, Oct.-Dec. 1979.

[14] Q. G. Wang, T. H. Lee, and C. Lin, Relay Feedback: Analysis, Identification and Control. London, U.K.: Springer, 2003.

[15] E. Colinet, J. Juillard, L. Nicu, and C. Bergaud, "Digital self-calibration method for MEMS sensors," IEEE Trans. Instrum. Measur., vol. 54, no. 4, pp. 1438-1443, Aug. 2005.

[16] O. Feely and L. O. Chua, "The effect of integrator leak in sigma-delta modulation," IEEE Trans. Circuits Syst. I, Fundam. Theory Appl., vol. 38, no. 11, pp. 1294-1305, Nov. 1991.

[17] V. Friedman, "The structure of the limit cycles in sigma-delta modulation," IEEE Trans. Commun., vol. 36, no. 8, pp. 972-979, Aug. 1988.

[18] J. Z. Tsypkin, Théorie des asservissements par plus-ou-moins. Paris: Dunod, 1962.

[19] [please update, volume, issue number, pages??]B. Hamel, "Contribution à l'étude mathématique des systèmes de réglage par tout-ou-rien," Centre d'études de mécanique du vol, Service Technique Aéronautique, no. 17, 1949.

[20] Chronos, La mesure de la fréquence des oscillateurs. Paris: Masson, 1991.

[21] R. C. Titsworth, "Equivalence classes of periodic sequences," Illinois J. Mathematics, vol. 8, pp. 266-270, 1964.

[22] Combinatorial Object Server [Online]. Available: http://www.theory. csc.uvic.ca/ cos/root.html

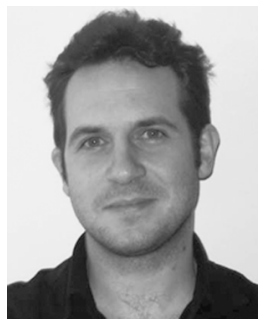

Jérôme Juillard was born in Nice, France, in 1973. He received the engineer degree from the École Centrale de Paris (ECP), Paris, France, with a specialization in electronics and signal processing, the Master's degree from IRCAM, Paris, France, and the Ph.D. degree in physical acoustics from University Paris 7, Paris, France, in 1995, [AU: YEAR?], and 1999, respectively

He worked at the Non-Destructive Testing Department, French Energy Commission, Paris, France, while working on his doctoral thesis. In 2000, he joined the Department of Signal Processing and Electronic Systems, SUPELEC, Paris, France, where he now is an Associate Professor. His current research interests include resonant nonlinear systems, and design and simulation of nonlinear, multiphysics problems, as they appear in microelectromechanical systems.

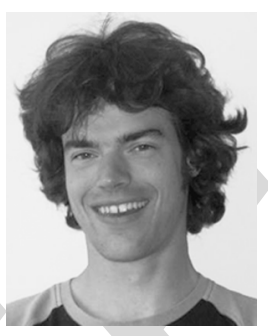

Eric Colinet was born in Mont Saint-Aignan, France, in 1979. He received the electrical engineer degree from the National Institute of Applied Sciences (INSA), Lyon, France, in 2002 the M.Sc. degree from [AU: WHICH INSTITUTION?] in microelectronics, in 2002, and the Ph.D. degree from the University of Paris 11, Paris, France, in 2005.

He worked on his Ph.D. thesis in the Department of Signal Processing and Electronic Systems of SUPELEC, Paris, France. He is currently a Research Engineer for the French Atomic Energy Commission, Laboratory of Microelectronics and Technology for Information (CEA-LETI), Grenoble, France. His work focuses on the development of novel microelectromechanical systems architectures, mostly in the field of resonant sensors and of delta-sigma sensors. 